\title{
On the Oscillation of Impulsive Partial Fractional Differential Equations
}

\author{
Zhuo Qu, Siying Zhu, Meng Su and Anping Liu* \\ School of Mathematics and Physics, China University of Geosciences, Wuhan, Hubei, 430074, China \\ ${ }^{*}$ Corresponding author
}

\begin{abstract}
In this paper, we investigate the oscillation properties of a class of impulsive partial fractional differential equations with several delays. Some sucient conditions for oscillation of the solutions are obtained by employing integral transformation technique and differential inequality method, and an example is given to illustrate the main result.
\end{abstract}

Keywords-oscillation; impulsive; delays; partial fractional differential equations

\section{INTRODUCTION}

It is well known that many important mathematical models are described by differential equations containing fractional order derivatives. The theories of fractional differential equations and their applications have been investigated extensively, we can find numerous applications in viscoelasticity, electrochemistry, control, porousmedia, electromagnetic, etc. see[1-6]. The oscillatory behavior of ordinary differential equations, partial differential equations and impulsive partial differential equations have been investigated by many papers in the past, see [7-11]. The oscillatory behavior of various classes of fractional ordinary differential equations and fractional partial differential equations have been investigated by many authors in recent years, see[12-20].

However, to the best of our knowledge very little is known regarding the oscillatory behavior of impulsive partial fractional differential equations with several delays up to now. Recently, A. Raheem and Md. Maqbul studied the oscillation behavior of solutions of a class of impulsive partial fractional differential equations with forcing term subject to Robin and Dirichlet boundary conditions by using differential inequality method in [21].

The purpose of this paper is to study the oscillation properties of the solutions to a class of impulsive partial fractional differential equations with several delays

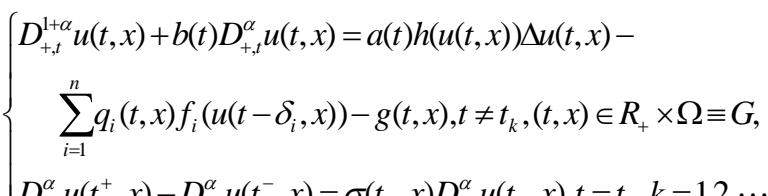

with the boundary condition

$$
\frac{\partial u(t, x)}{\partial n}=w(t, x, u(t, x)),(t, x) \in R_{+} \times \partial \Omega, t \neq t_{k} .
$$

Where $\alpha \in(0,1)$ is a constant; $D_{+, t}^{\alpha}$ is the RiemannLiouville fractional derivative of order $\alpha$ of $u(t, x)$ with respect to $t ; \Omega$ is a boundary domain in $R^{n}$ with a smooth boundary $\partial \Omega$ and $\bar{\Omega}=\Omega \cup \partial \Omega ; R_{+}=(0,+\infty), \Delta$ is Laplacian operator, and $n$ is the unit exterior normal vector to $\partial \Omega$; $b(t), \mathrm{a}(\mathrm{t}) \in \mathrm{PC}\left[R_{+}, R_{+}\right]$, PC denote the class of functions which are piecewise continuous in $t$ with discontinuities of first kind only at $t=t_{k} ; \delta_{i}=$ const. $>0$. The solution $u(t, x)$ of the problem (1) - (2) and $D_{+, t}^{\alpha} u(t, x)$ are piecewise continuous with discontinuities of first kind only at $t=t_{k}$, and left continuous at $t=t_{k}, k=1,2, \cdots$.

Throughout this paper, we assume that the following conditions hold:

(H1): $f_{i}: R \rightarrow R$ is a continuous function such that $f_{i}(u) / u \geq k_{i}>0$, for all $u \neq 0$; and $k_{i}$ is a positive constant.

(H2): $q_{i}(t, x) \in P\left[R_{+} \times \bar{\Omega}, R_{+}\right]$, and $q_{i}(t)=\min _{1 \leq i \leq n} \min _{x \in \bar{\Omega}} q_{i}(t, x)$.

(H3): $g(t, x) \in P C\left[R_{+} \times \bar{\Omega}, R\right]$.

(H4): $\mathrm{h}(\mathrm{u}) \in \mathrm{C}(\mathrm{R}, \mathrm{R})$; $\mathrm{uh}^{\prime}(\mathrm{u}) \geq 0 ; \mathrm{w}(\mathrm{t}, \mathrm{x}, \mathrm{u})$ is a piecewise continous function, such that $u w(t, x, u) h(u) \leq 0$.

(H5) $\sigma: R_{+} \times \bar{\Omega} \rightarrow R_{+}$such that $\sigma\left(t_{k}, x\right) \leq \alpha_{k}$.

(H6): The given numbers $0<t_{1}<\cdots<t_{k}<\cdots$, are such that $\lim _{k \rightarrow \infty} t_{k}=+\infty$.

(H7): At the moments of impulsive the following relation is satisfied

$$
D_{+, t}^{\alpha} u\left(t_{k}^{-}, x\right)=D_{+, t}^{\alpha} u\left(t_{k}, x\right) .
$$

For the sake of convenience, in this paper, we denote 


$$
U(t)=\int_{\Omega} u(t, x) d x, G(t)=\int_{\Omega} g(t, x) d x
$$

\section{PReliminaries}

\section{A. Definition 2.1}

A nonzero solution $u(t, x)$ of the problem (1)-(2) is said to be nonoscillatory in the domain $G$ if there exists a number $t_{0} \geq 0$ such that $u(t, x)$ has a constant sign for $(t, x) \in\left[t_{0},+\infty\right) \times \Omega$. Otherwise, it is said to be oscillatory.

\section{B. Definition 2.2}

The Riemann-Liouville fractional partial derivative of order $\alpha>0$ with respect to $t$ of a function $u(t, x)$ on the half-axis $R_{+}$is defined by

$$
D_{+, t}^{\alpha} u(t, x)=\frac{1}{\Gamma(1-\alpha)} \frac{\partial}{\partial t} \int_{0}^{t}(t-v)^{-\alpha} u(v, x) d v, t>0
$$

where $\alpha \in(0,1) ; \Gamma \quad$ is gamma function, and $\Gamma(\alpha)=\int_{0}^{+\infty} s^{\alpha-1} e^{-s} d s$.

\section{Definition 2.3}

The Riemann-Liouville fractional derivative of order $\alpha>0$ of a function $x: R_{+} \rightarrow R$ on the half-axis $R_{+}$is defined by

$$
\left.D_{+, t}^{\alpha} x(t)=\frac{1}{\Gamma(\lceil\alpha\rceil-\alpha)} \frac{d^{\lceil\alpha\rceil}}{d t^{[\alpha\rceil}} \int_{0}^{t}(t-v)\right)^{\lceil\alpha\rceil-\alpha-1} x(v) d v, t>0,
$$

where $\alpha \in(0,1) ; \Gamma$ is gamma function, $\lceil\alpha\rceil$ is the ceiling function of $\alpha$.

\section{Lemma $2.4^{[3]}$}

Let $0<\alpha<1, m \in N$ and $D=d / d x$. If the fractional derivatives $\left(D_{+}^{\alpha} y\right)(x)$ and $\left(D_{+}^{m+\alpha} y\right)(x)$ exist, then

$$
\left(D^{m} D_{+}^{\alpha} y\right)(x)=\left(D_{+}^{m+\alpha} y\right)(x)
$$

\section{MAIN RESULTS}

A. Theorem 3.1

If impulsive fractional differential inequality

$$
D_{+, t}^{1+\alpha} U(t)+b(t) D_{+, t}^{\alpha} U(t) \leq-G(t)
$$

$$
D_{+, t}^{\alpha} U\left(t^{+}\right) \leq\left(1+\alpha_{k}\right) D_{+, t}^{\alpha} U(t), k=1,2, \cdots,
$$

has no eventually positive solutions and impulsive fractional differential inequality

$$
\begin{gathered}
D_{+, t}^{1+\alpha} U(t)+b(t) D_{+, t}^{\alpha} U(t) \geq-G(t), \\
D_{+, t}^{\alpha} U\left(t^{+}\right) \geq\left(1+\alpha_{k}\right) D_{+, t}^{\alpha} U(t), k=1,2, \cdots,
\end{gathered}
$$

has no eventually negative solutions, then every nonzero solution $u(t, x)$ of the problem (1) and (2) is oscillatory in the domain $G$.

Proof. Suppose to contrary that $u(t, x)$ be a nonzero solution of the problem (1) and (2) which is nonoscillatory in the domain $G$. Without loss of generality, we assume that $u(t, x)$ is an eventually positive solution of problem (1) and (2) in the domain $G$, then there exists a $t_{0} \geq 0$ such that $u(t, x)>0, u\left(t-\delta_{i}\right)>0$, for $(t, x) \in\left[t_{0},+\infty\right) \times \Omega$.

Case 1: $t \neq t_{k}$. Integrating the first equation of problem (1) with respect to $x$ over the domain $\Omega$, we have

$$
\begin{aligned}
& \int_{\Omega} D_{+, t}^{1+\alpha} u(t, x) d x+b(t) \int_{\Omega} D_{+, t}^{\alpha} u(t, x) d x= \\
& a(t) \int_{\Omega} h(u(t, x)) \Delta u(t, x) d x-\int_{\Omega} \sum_{i=1} q_{i}(t, x) f_{i}\left(u\left(t-\delta_{i}, x\right)\right) d x \\
& -\int_{\Omega} g(t, x) d x
\end{aligned}
$$

By using Green's formula, combing boundary condition (2) and assumption (H4), we obtain

$$
\begin{aligned}
& \int_{\Omega} h(u) \Delta u(t, x) d x=\int_{\partial \Omega} h(u) \frac{\partial u(t, x)}{\partial n} d x-\int_{\Omega} h^{\prime}(u)|\operatorname{gradu}|^{2} d x \\
& =\int_{\partial \Omega} h(u) w(t, x, u) d x-\int_{\Omega} h^{\prime}(u)|\operatorname{gradu}|^{2} d x \leq 0
\end{aligned}
$$

According to assumption (H1) and (H2), we have

$$
\begin{aligned}
& \int_{\Omega} \sum_{i=1}^{n} q_{i}(t, x) f_{i}\left(u\left(t-\delta_{i}, x\right)\right) d x \\
& \geq \sum_{i=1}^{n} k_{i} q_{i}(t) \int_{\Omega} u\left(t-\delta_{i}, x\right) d x \geq 0, t \geq t_{0} .
\end{aligned}
$$

And according to Lemma 2.4, combing (11)-(13) , we can easily obtain 


$$
\begin{aligned}
& D_{+, t}^{1+\alpha} U(t)+b(t) D_{+, t}^{\alpha} U(t) \leq-\sum_{\mathrm{i}=1}^{\mathrm{n}} k_{i} q_{i}(t) \int_{\Omega} u\left(t-\delta_{i}, x\right) d x \\
& \leq-\sum_{\mathrm{i}=1}^{\mathrm{n}} k_{i} q_{i}(t) \int_{\Omega} u\left(t-\delta_{i}, x\right) d x-G(t) \leq-G(t), t \geq t_{0} .
\end{aligned}
$$

Case 2: $t=t_{k}$.Integrating the second equation of problem (1) with respect to $x$ over the domain $\Omega$, and according to assumption (H5), we have

$$
\begin{aligned}
& D_{+, t}^{\alpha} U\left(t_{k}^{+}\right)=D_{+, t}^{\alpha} \int_{\Omega} u\left(t_{k}^{+}, x\right) d x \\
& \leq\left(1+\alpha_{k}\right) D_{+, t}^{\alpha} \int_{\Omega} u\left(t_{k}, x\right) d x=\left(1+\alpha_{k}\right) D_{+, t}^{\alpha} U\left(t_{k}\right) .
\end{aligned}
$$

Thus impulsive fractional differential inequality (14) and (15) imply that the function $U(t)=\int_{\Omega} u(t, x) d x$ is an eventually positive solution of the fractional impulsive differential inequality (7) and (8) which contradicts the conditions of the theorem.

On the other hand, if $u(t, x)$ is an eventually negative solution of the problem (1) and (2) in the domain $G$, then using the similar method, we can easily obtain that $U(t)=\int_{\Omega} u(t, x) d x$ is an eventually negative solution of the fractional impulsive differential inequality (9) and (10) which contradicts the conditions of the theorem. The proof is completed.

\section{B. Lemma $3.2^{[4]}$}

Assume that

$$
\begin{aligned}
& \omega^{\prime}(t) \leq g_{1}(t) \omega(t)+g_{2}(t), t \neq t_{k}, t \geq t_{0}, \\
& \omega\left(t_{k}^{+}\right) \leq\left(1+a_{k}\right) \omega\left(t_{k}\right), k=1,2, \cdots,
\end{aligned}
$$

where $0<t_{1}<\cdots<t_{k}<\cdots$ and $\lim _{k \rightarrow \infty}=+\infty ; \omega \in P C^{1}\left[R_{+}, R\right]$; $g_{1}, g_{2} \in\left[R_{+}, R\right]$ and $a_{k}$ are constants. Then

$$
\begin{aligned}
\omega(t) \leq & \omega\left(t_{0}\right) \prod_{t_{0}<t_{l}<t}\left(1+a_{k}\right) \exp \left(\int_{t_{0}}^{t} g_{1}(s) d s\right) \\
& +\int_{t_{0}}^{t} \prod_{s<t_{l}<t}\left(1+a_{k}\right) \exp \left(\int_{s}^{t} g_{1}(\sigma) d \sigma\right) g_{2}(s) d s
\end{aligned}
$$

\section{Lemma $3.3^{[4]}$}

If $U(t)$ is a solution of the impulsive fractional differential inequality (7) and (8) (or (9) and (10)), and

$$
E(t)=\int_{0}^{t}(\tau-t) U(\tau) d \tau, \phi \circ \rho \alpha \in(0,1), \alpha v \delta t>0,
$$

then

$$
E^{\prime}(t)=\Gamma(1-\alpha)\left(D_{+, t}^{\alpha} U\right)(t), \phi \circ \rho \alpha \in(0,1), \alpha \vee \delta t>0 .
$$

\section{Theorem 3.4}

If for some $\tau_{2}>0$,

$$
\int_{\tau_{2}}^{\infty} \exp (-b(\sigma) d \sigma) d s=\infty
$$

further, for some $\tau_{1} \geq 0$,

$$
\limsup _{t \rightarrow \infty} \frac{\int_{\tau_{1}}^{t} \prod_{s<t_{1}<t}\left(1+\alpha_{k}\right) \exp \left(-\int_{s}^{t} b(\sigma) d \sigma\right) G(s) d s}{\prod_{\tau_{1}<t_{1}<t}\left(1+\alpha_{k}\right) \exp \left(-\int_{\tau_{1}}^{t} b(s)\right) d s}=\infty
$$

and

$$
\liminf _{t \rightarrow \infty} \frac{\int_{\tau_{1}}^{t} \prod_{s<t_{1}<t}\left(1+\alpha_{k}\right) \exp \left(-\int_{s}^{t} b(\sigma) d \sigma\right) G(s) d s}{\prod_{\tau_{1}<t_{1}<t}\left(1+\alpha_{k}\right) \exp \left(-\int_{\tau_{1}}^{t} b(s)\right) d s}=-\infty,
$$

then each nonzero solution of problem (1) and (2) is oscillatory in the domain $G$.

Proof. To prove the theorem, it is sufficient to prove that the impulsive fractional differential inequality (7) and (8) has no eventually positive solutions, and the impulsive fractional differential inequality (9) and (10) has no eventually negative solutions. Suppose to contrary that the impulsive fractional differential inequality (7) and (8) has an eventually positive solution $U(t)$, then there exists $\tau_{1} \geq 0$ such that $U(t)>0$, $U\left(t-\delta_{i}\right)>0, G(t)>0, t \geq \tau_{1}$.

Let

$$
v(t, x)=\exp \left(\int_{t_{0}}^{t} b(s) d s\right)
$$

By using Lemma 2.4 and the impulsive fractional differential inequality (7), we have

$$
\begin{aligned}
& {\left[\left(D_{+,}^{\alpha}{ }_{t} U\right) v(t)\right]^{\prime}=\left(D_{+,}^{\alpha}{ }_{t} U\right)^{\prime}(t) v(t)+b(t)\left(D_{+,}^{\alpha}{ }_{t} U\right)(t) v(t)} \\
& \leq-G(t) v(t)<0
\end{aligned}
$$

Thus $\left(D_{+}^{\alpha},{ }_{t} U\right)(t) v(t)$ is strictly decreasing for $t \geq \tau_{1}$ and is eventually of constant sign. Since $v(t)>0$, for $t \geq \tau_{1}$, we know that $\left(D_{+,{ }_{t}}^{\alpha} U\right)(t) \quad$ is eventually of constant sign. Then, $\left(D_{+,}^{\alpha}{ }_{t} U\right)(t)>0$ for $t \geq \tau_{1}$. Otherwise, $\left(D_{+,}^{\alpha}{ }_{t} U\right)(t)<0$ for $t \geq \tau_{1}$, 
there exists $\tau_{2} \in\left[\tau_{1}, \infty\right)$, such that $\left(D_{+,}^{\alpha}{ }_{t} U\right)\left(\tau_{2}\right) v\left(\tau_{2}\right)<0$. Since $\left(D_{+, t}^{\alpha} U\right)(t) v(t)$ is strictly decreasing for $t \geq \tau_{1}$, so $\left(D_{+,}^{\alpha}{ }_{t} U\right)(t) v(t)<\left(D_{+,}^{\alpha}{ }_{t} U\right)\left(\tau_{2}\right) v\left(\tau_{2}\right)=C<0$ for $t \geq \tau_{2}$. From Lemma 3.3 , we have

$$
\frac{E^{\prime}(t)}{\Gamma(1-\alpha)}=\left(D_{+,}^{\alpha} U\right)(t)<C \exp \left(-\int_{t_{0}}^{t} b(s) d s\right), t \geq \tau_{2} .
$$

Integrating from $\tau_{2}$ to $t$, we have

$$
E(t)<E\left(\tau_{2}\right)+\Gamma(1-\alpha) C \int_{\tau_{2}}^{t} \exp \left(-\int_{t_{0}}^{t} b(\sigma) d \sigma\right) d s
$$

Letting $t \rightarrow \infty$, and using condition (18), we get $\lim _{t \rightarrow \infty} E(t)=-\infty$, which is controdiction to the fact that $E(t)>0$. Hence $\left(D_{+, t}^{\alpha} U\right)(t)>0$ for $t \geq \tau_{1}$. Let $\omega(t)=\left(D_{+, t}^{\alpha} U\right)(t)$. From Lemma 2.4 and impulsive fractional inequality (7) and (8), we have

$$
\begin{aligned}
& \omega^{\prime}(t) \leq-b(t) \omega(t)-G(t), t \geq t_{0}, t \neq t_{k}, \\
& \omega\left(t_{k}^{+}\right) \leq\left(1+\alpha_{k}\right) \omega\left(t_{k}\right) \cdot k=1,2, \cdots .
\end{aligned}
$$

By using Lemma 3.2, we have

$$
\begin{aligned}
\omega(t) & \leq \omega\left(\tau_{1}\right) \prod_{\tau_{1}<t_{1}<t}\left(1+\alpha_{k}\right) \exp \left(-\int_{\tau_{1}}^{t} b(s) d s\right) \\
& -\int_{\tau_{1}}^{t} \prod_{s<t_{1}<t}\left(1+\alpha_{k}\right) \exp \left(-\int_{s}^{t} b(\sigma) d \sigma\right) G(s) d s
\end{aligned}
$$

by using the condition (19) and taking $t \rightarrow \infty$, it follows from above equation

$$
\liminf _{t \rightarrow \infty} \frac{\int_{\tau_{1}}^{t} \prod_{s<t_{1}<t}\left(1+\alpha_{k}\right) \exp \left(-\int_{s}^{t} b(\sigma) d \sigma\right) G(s) d s}{\prod_{\tau_{1}<t_{1}<t}\left(1+\alpha_{k}\right) \exp \left(-\int_{\tau_{1}}^{t} b(s)\right) d s}=-\infty
$$

which contradicts $\omega(t)>0$. The proof is completed.

On the other hand, suppose to contrary that the impulsive fractional differential inequality (9) and (10) has an eventually negative solution $U(t)$, then there exists $\tau_{1} \geq 0$ such that $U(t)<0, U\left(t-\delta_{i}\right)<0, G(t)<0, t \geq \tau_{1}$. Then using similar methods, according the condition (20) and taking $t \rightarrow \infty$, we can obtain

$$
\limsup _{t \rightarrow \infty} \frac{\int_{\tau_{1}}^{t} \prod_{s<t_{1}<t}\left(1+\alpha_{k}\right) \exp \left(-\int_{s}^{t} b(\sigma) d \sigma\right) G(s) d s}{\prod_{\tau_{1}<t_{1}<t}\left(1+\alpha_{k}\right) \exp \left(-\int_{\tau_{1}}^{t} b(s)\right) d s}=\infty,
$$

which contradicts $\omega(t)<0$. The proof is completed.

\section{EXAMPLE}

In this section, we discuss an illustrative example.

Example 4.1 Consider the following problem

$\left\{\begin{array}{l}D_{+, t}^{\frac{7}{4}} u(t, x)+\frac{1}{t} D_{+, t}^{\frac{3}{4}} u(t, x)=e^{-t} u^{2}(t, x) \Delta u(t, x)- \\ \left(1+t^{2}+x^{2}\right) u\left(t-\frac{2 \pi}{3}, x\right) e^{\left[u\left(t-\frac{2 \pi}{3}, x\right)\right]^{2}}-t \cos x, t \neq t_{k}, \\ D_{+, t}^{\frac{3}{4}} u\left(t_{k}^{+}, x\right)-D_{+, t}^{\frac{3}{4}} u\left(t_{k}^{-}, x\right)=t_{k}^{-3} \cos x D_{+, t}^{\frac{3}{4}} u\left(t_{k}, x\right), t=t_{k} .\end{array}\right.$

with the boundary condition

$\frac{\partial u(t, x)}{\partial n}=w(t, x, u(t, x))=-u^{3}(t, x),(t, x) \in R_{+} \times \partial \Omega, t \neq t_{k}$

Here

$\alpha=\frac{3}{4}, \Omega=\left(0, \frac{\pi}{2}\right), n=1 . b(t)=\frac{1}{t}, a(t)=e^{-t}, h(u)=u^{2}$,

$$
\begin{gathered}
q_{1}(t, x)=1+t^{2}+x^{2}, f_{1}(u)=u e^{u^{2}}, \delta_{1}=\frac{2 \pi}{3}, g(t, x)=t \cos x, \\
\sigma\left(t_{k}, x\right)=t_{k}^{-3} \cos x, \alpha_{k}=k_{k}^{-3},(t, x) \in R_{+} \times\left(0, \frac{\pi}{2}\right) .
\end{gathered}
$$

We can easily see that

$$
\int_{\tau_{2}}^{\infty} \exp (-b(\sigma) d \sigma) d s=\int_{\tau_{2}}^{\infty} \exp \left(-\int_{t_{0}}^{t} \frac{1}{\sigma} b(\sigma) d \sigma\right) d s=\int_{\tau_{2}}^{\infty} \frac{t_{0}}{t} d t=\infty,
$$

$$
\limsup _{t \rightarrow \infty} \frac{\int_{\tau_{1}}^{t} \prod_{s<t_{1}<t}\left(1+\alpha_{k}\right) \exp \left(-\int_{s}^{t} b(\sigma) d \sigma\right) G(s) d s}{\prod_{\tau_{1}<t_{1}<t}\left(1+\alpha_{k}\right) \exp \left(-\int_{\tau_{1}}^{t} b(s)\right) d s}=\infty
$$

and 


$$
\liminf _{t \rightarrow \infty} \frac{\int_{\tau_{1}}^{t} \prod_{s<t_{1}<t}\left(1+\alpha_{k}\right) \exp \left(-\int_{s}^{t} b(\sigma) d \sigma\right) G(s) d s}{\prod_{\tau_{1}<t_{1}<t}\left(1+\alpha_{k}\right) \exp \left(-\int_{\tau_{1}}^{t} b(s)\right) d s}=-\infty .
$$

[21] A. Raheem, M. Maqbul, Oscillation criteria for impulsive partial fractional differential equations. Computers and Mathematics with Applications, 2017, 73(8):1781-1788.
The conditions of Theorem 3.4 are satisfied, thus every solution of the problem (21)-(22) oscillates.

\section{REFERENCES}

[1] I. Podlubny, Fractional Differential Equations. Academic Press, San Diego, 1999

[2] S.G. Samko, A.A. Kilbas, O.I. Marichev, Fractional Integrals and Derivatives: Theory and Applications. Elsevier, Amsterdam, 1993.

[3] A.A. Kilbas, H.M. Srivastava, J.J. Trujillo, Theory and Applications of Fractional Differential Equations. Elsevier Science BV, Amsterdam, 2006.

[4] V.Lakshmikantham, D.D. Bainov, P.S. Simeonov, Theory of Impulsive Differential Equations. World Scientific, Singapore, 1989.

[5] A. Saadatmandi, M. Dehghan, A new operational matrix for solving fractional-order differential equations. Computers and Mathematics with Applications, 2010, 59(3):1326-1336.

[6] Y.Zhou, Basic Theory of Fractional Differential Equations. World Scientific, Singapore, 2014.

[7] D.D. Bainov, E. Minchev, Oscillation of solutions of impulsive nonlinear parabolic differential-difference equations. International Journal of Theoretical Physics, 1996, 35(1):207-215.

[8] J.R. Yan, Oscillation properties of a second-order impulsive delay differential equation. Computers and Mathematics with Applications, 2004, 47(2):253-258

[9] A.p. Liu, L. Xiao, T. Liu, M. Zou, Oscillation of Nonlinear Impulsive Hyperbolic Equation with Several Delays, Rocky Mountain Journal of Mathematics, 2007, 37(2007):1669-1684.

[10] P. Prakash, S. Harikrishnan, Oscillation of solutions of impulsive vector hyperbolic differential equations with delays. Applicable Analysis, 2012, 91(3):459-473.

[11] Q.X. Ma, A.P. Liu, Oscillation criteria of neutral type impulsive hyperbolic equations, Acta Mathematica Scientia, 2014, 34(6):18451853.

[12] S.R. Grace, R.P. Agarwal, J.Y. Wong, A. Zafer, On the oscillation of fractional differential equations. Fractional Calculus and Applied Analysis, 2012, 15(2):222-231.

[13] D.X. Chen, Oscillation criteria of fractional differential equations. Advances in Difference Equations, 2012, 2012(1):33.

[14] Q.H. Feng, F.W. Meng, Oscillation of solutions to nonlinear forced fractional differential equations. Electronic Journal of Differential Equations, 2013, 2013(169):1471-1484.

[15] P.Prakash, S.Harikrishnan, J.J. Nieto, J.H. Kim, Oscillation of a time fractional partial differential equation. Electronic Journal of Qualitative Theory of Differential Equations, 2014, 15(11):1-10.

[16] S. Harikrishnan, P. Prakash, J.J. Nieto, Forced oscillation of solutions of a nonlinear fractional partial differential equation. Applied Mathematics and Computation, 2015, 254(254):14-19.

[17] W.N. Li, On the forced oscillation of certain fractional partial differential equations. Applied Mathematics Letters, 2015, 50:5-9.

[18] M. Bayram, H. Adiguzel, A. Secer, Oscillation criteria for nonlinear fractional differential equation with damping term. Open Physics, 2016, 14(1):119-128.

[19] J.C. Yang, A.P. Liu, T. Liu, Forced oscillation of nonlinear fractional differential equations with damping term, Advances in Difference Equations, 2015, 2015(1):1.

[20] Y.F. Xiong, S.Y. Zhu, A.P. Liu, On the Forced Oscillation of Fractional Delay Paitial Differential Equations. Journal of Biomathematics, 2017. 32(3):304-310. 